\title{
Caracterización de la Regulación Metacognitiva en la Resolución de Problemas sobre Medidas de Tendencia Central
}

\section{The Characterization of Metacognitive Regulation in the Resolution of Central Tendency Problems}

\author{
Amaury de Jesús \\ 'Universidad Arturo Prat, Programa de Doctorado en Ciencias de la Educación, Iquique, Chile. \\ E-mail: aarrieta@estudiantesunap.cl
}

Resumen: La resolución de problemas es un tema importante dentro de las matemáticas, debido a que permite a los estudiantes aplicar lo que aprenden a situaciones de la vida cotidiana. Es por esto, que esta investigación tiene por objetivo caracterizar de qué manera la regulación metacognitiva favorece los procesos de resolución de problemas sobre medidas de tendencia central, presenta un enfoque cualitativo con carácter descriptivo, y el diseño metodológico es un estudio de caso, en el cual se analizan 6 estudiantes del grado $8^{\circ}$. Los resultados muestran como la planeación, el monitoreo y la evaluación, permiten a los estudiantes ser más organizados, identificar y corregir errores, saber por qué realizan determinadas acciones y tener control de su propio aprendizaje. Además, se muestra cómo los obstáculos ontológicos que presentan los educandos en la resolución de problemas están relacionados con sus concepciones, influenciadas por la utilización de términos en el lenguaje cotidiano.

Palabras clave: Enseñanza de las matemáticas; Resolución de problemas; Metacognición; Aprendizaje.

Abstract: Problem solving is an important topic in mathematics because it allows students to apply what they learn to situations of everyday life. This is why this research aims to characterize how metacognitive regulation favors the processes of solving problems of measures of central tendency. The qualitative approach has a descriptive character, and the methodological design is a case study in which six 8th-grade students are analyzed. The results show how planning, monitoring and evaluation allow students to be more organized, identify and correct errors, know why they perform certain actions, and have control over their own learning. In addition, it is shown how the ontological obstacles demonstrated by learners in the resolution of problems are related to their conceptions, which are influenced by the use of everyday language.

Keywords: Mathematics teaching; Problem solving; Metacognition. Learning.

Recebido em: 29/10/2019

Aprovado em: 01/04/2020 


\section{Introducción}

La resolución de problemas es considerada una parte esencial de la educación matemática, debido a que por medio de esta, los estudiantes aplican lo aprendido en situaciones de la vida diaria, además, permite que se pongan en práctica una serie de habilidades como razonar, inferir, comunicar, crear, analizar; y de esta manera poder enfrentarse a diferentes escenarios de la cotidianidad. En este sentido, Orton (1990, p. 51) plantea que "[...] la resolución de problemas puede considerarse como la verdadera esencia de las matemáticas". Sin embargo, los estudiantes son poco reflexivos cuando resuelven problemas, y no se detienen a analizar las dificultades en el proceso de resolución; a comprender los pasos realizados, a corregir los errores, a evaluar su desempeño, a regular su aprendizaje; presentando dificultades asociadas con la falta de comprensión de los enunciados, y en algunas ocasiones realizan razonamientos incorrectos, manifestando confusiones en la elección de las operaciones que deben realizar y su respectiva ejecución, están más orientados a resolver los problemas de forma mecánica, sin darle un sentido lógico a lo que están realizando.

Teniendo en cuenta lo anterior, esta investigación cobra importancia, debido a que permite caracterizar de qué manera la regulación metacognitiva favorece el proceso de resolución de problemas sobre medidas de tendencia central, logrando que los estudiantes se enfrenten a los problemas de una forma diferente, reflexionen sobre los métodos de solución, realicen monitoreo de sus estrategias y tengan control sobre su aprendizaje. El poder reconocer cómo los procesos metacognitivos están relacionados con ciertas características de la resolución de problemas, brinda información pertinente a los docentes para desarrollar estrategias didácticas que permitan mejorar los niveles de desempeño y destrezas de los estudiantes al momento de resolver problemas.

De igual manera, el presente texto muestra los obstáculos ontológicos y procedimentales que presentan los estudiantes cuando solucionan problemas sobre medidas de tendencia central, el cual son un referente necesario para plantear acciones que permitan superar las dificultades de los educandos. Así mismo, en esta investigación se diseña y aplica una unidad didáctica que contiene actividades y preguntas metacognitivas que permiten verificar los procesos de planeación, monitoreo y evaluación que realizan los estudiantes cuando resuelven problemas y de esta manera conocer y regular sus acciones.

\section{Marco Teórico}

Los antecedentes que se presentan a continuación dan cuenta de los resultados de algunas investigaciones que reconocen la importancia de la resolución de problemas y metacognición en matemáticas.

Mato-Vázquez, Espiñeira, y López-Chao (2017) realizaron una investigación en la cual analizaron las implicaciones que tiene la incorporación de estrategias metacognitivas en el aprendizaje matemático con estudiantes de sexto grado de educación primaria. Los resultados muestran, tras una prueba de diagnóstico (pretest) y otra de referencia (postest), mejoras en atención, comprensión, trabajo cooperativo, resolución de problemas, procesos de aprendizaje, confianza y motivación. La utilización de estrategias metacognitivas permite que el estudiante controle la comprensión, detecte errores y examine los saberes previos. 
Ortiz Bueno y Valencia Castrillón (2017) realizaron una investigación de tipo cualitativa el cual tuvo como objetivo reconocer las manifestaciones del conocimiento metacognitivo que se evidencian en estudiantes del grado quinto de básica primaria en la asignatura de matemáticas. Dentro de los resultados, se resaltan respecto al conocimiento de sí mismas, las 6 estudiantes analizadas evidenciaron ansiedad matemática, procesos de reflexión metacognitiva, y reconocimiento de dificultades. En relación al conocimiento de la tarea, algunas reflejaron conocimientos previos, y otras, carencia de conocimiento metacognitivo de la tarea. En cuanto al conocimiento de la estrategia, las alumnas manifestaron conocer la importancia de leer y releer.

Moreno Castiblanco y Daza Torres (2014) realizaron un proyecto de investigación en la que se utilizó como herramienta principal para el aprendizaje la metacognición. Se buscó determinar el impacto de diferentes estrategias metacognitivas en la resolución de problemas matemáticos en tres estudiantes de $7^{\circ}$ grado. Los resultados mostraron que los estudiantes desarrollaron de manera significativa procesos de planeación, los cuales les permitieron la toma de conciencia necesaria para poner en acción diversos mecanismos de solución de los diferentes problemas.

Pulido (2014) realizó una investigación que buscó caracterizar los procesos metacognitivos que llevan a cabo los estudiantes de grado noveno con desempeños superior y bajo en matemáticas cuando resuelven problemas relacionados con las operaciones básicas: suma, resta, multiplicación y división. El enfoque de investigación fue cualitativo con un alcance descriptivo. Se pudo concluir que los procesos metacognitivos no tienen una secuencia lineal, sino por el contrario, su relación es casi de superposición, en donde cada uno influencia al otro y se realizan de manera simultánea: mientras se planea se regula y evalúa, mientras se regula también se vuelve a planear y se evalúa nuevamente y así sucesivamente.

Quintero García (2014) realizó una investigación de tipo cualitativa el cual tuvo como objetivo reconocer las dificultades que identifican los estudiantes a través de la metacognición en el aprendizaje de las matemáticas. Dentro de los resultados se destaca que la dificultad para recordar y la falta de atención en clase parecen estar asociadas al fracaso durante la solución de problemas en contextos matemáticos, en todos los niveles de la secundaria, además, no entender el problema es frecuente en casi todos los grados y está comúnmente relacionado con la falta de análisis y a su vez con deficiencias en la comprensión lectora.

Teniendo en cuenta los antecedentes expuestos anteriormente, se puede concluir que la implementación de la reflexión metacognitiva mejora los procesos de enseñanzaaprendizaje y ayuda a los estudiantes a resolver problemas de una forma organizada, caracterizándose por la toma de conciencia, en la cual, el educando es capaz de planear, monitorear y controlar el proceso mental de sí mismo. De esta manera, se presenta un proceso de reflexión al momento de seleccionar las estrategias e identificar los errores, para tenerlos en cuenta en la resolución de problemas posteriores. Del mismo modo, para que la metacognición sea efectiva, se debe ceder progresivamente el control a los estudiantes, dando una guía inicial, permitiendo generar autonomía en ellos, logrando procesos de reflexión sobre el trabajo que realicen. 


\section{Resolución de problemas}

Lesh y Zawojewski (2007, p. 782) definen la resolución de problemas como

[...] el proceso de interpretar una situación matemáticamente, la cual involucra varios ciclos interactivos de expresar, probar y revisar interpretaciones, de ordenar, integrar, modificar, revisar o redefinir grupos de conceptos matemáticos desde varios tópicos dentro y más allá de las matemáticas.

En este sentido, el estudiante para resolver problemas debe realizar un proceso con diferentes pasos que le permitan recolectar, organizar y analizar información, aplicar estrategias para poder llegar a la solución.

\section{Factores que intervienen en el proceso de resolución de problemas matemáticos}

Schoenfeld (1992), presenta una caracterización de las dimensiones o categorías que explican el éxito o fracaso de los estudiantes en la resolución de problemas:

a. El conocimiento o recursos básicos que incluye definiciones, hechos, formulas, algoritmos y conceptos fundamentales asociados con un dominio matemático particular o tema.

b. Estrategias cognitivas o heurísticas que involucran formas de representar y explorar los problemas con la intención de comprender los enunciados y plantear caminos de solución. Algunos ejemplos de estas estrategias son dibujar un diagrama, buscar un problema análogo, establecer submetas, descomponer el problema, etc.

c. Las estrategias metacognitivas que involucran conocimiento acerca del funcionamiento cognitivo propio del individuo (¿Qué necesito? ¿Cómo utilizo ese conocimiento?) y estrategias de monitoreo y control del propio proceso cognitivo (¿Qué estoy haciendo? ¿Por qué lo hago? ¿A dónde voy?).

d. Las creencias y componentes afectivos que caracterizan la conceptualización del individuo acerca de las matemáticas y la resolución de problemas, y la actitud y disposición a involucrarse en actividades matemáticas.

\section{La Metacognición}

La metacognición se refiere, según, $\operatorname{Klinger}(2001$, p. 85), como "[...] el conocimiento que el individuo tiene sobre los procesos de cognición y de estados tales como la memoria, la atención, el conocimiento, la conjetura y la ilusión".

La regulación metacognitiva, según Schraw (1998 apud TAMAYO, 2006, p. 277), se refiere al conjunto de actividades que ayudan al estudiante a controlar su aprendizaje; se relaciona con las decisiones del aprendiz antes, durante y después de realizar cierta tarea de aprendizaje. Se asume que la regulación metacognitiva mejora el rendimiento en diferentes formas: mejora el uso de la atención, proporciona una mayor conciencia de las dificultades en la comprensión y mejora las estrategias ya existentes. Se ha encontrado un incremento significativo del aprendizaje cuando se incluyen, como parte de la enseñanza, la regulación y la comprensión de las actividades. 
Brown (1987 apud TAMAYO, 2006, p. 277) señala los tres procesos cognitivos esenciales:

- Planeación: Es un proceso que se realiza antes de enfrentar una tarea o meta escolar, implica la selección de estrategias apropiadas y la localización de factores que afectan el rendimiento; la predicción, las estrategias de secuenciación y la distribución del tiempo o de la atención selectiva antes de realizar la tarea; consiste en anticipar las actividades, prever resultados, enumerar pasos.

- Monitoreo: se refiere a la posibilidad que se tiene, en el momento de realizar la tarea, de comprender y modificar su ejecución, por ejemplo, realizar auto-evaluaciones durante el aprendizaje, para verificar, rectificar y revisar las estrategias seguidas.

- Evaluación: Realizada al final de la tarea, se refiere a la naturaleza de las acciones y decisiones tomadas por el aprendiz; evalúa los resultados de las estrategias seguidas en términos de eficacia.

\section{Método}

\section{Enfoque metodológico}

Esta investigación tuvo un enfoque cualitativo con carácter descriptivo, debido a que además de conocer cuáles son las estrategias que utilizan los estudiantes para resolver problemas, se describe como la regulación metacognitiva favorece este proceso. En cuanto a la investigación cualitativa, Cortés Cortés e Iglesias León (2004, p. 10) manifiestan que:

Es una vía de investigar sin mediciones numéricas, tomando encuestas, entrevistas, descripciones, puntos de vista de los investigadores, reconstrucciones los hechos, no tomando en general la prueba de hipótesis como algo necesario, su alcance es más bien el de entender las variables que intervienen en el proceso más que medirlas y acotarlas.

\section{Diseño}

El diseño que se utilizó en esta investigación fue el estudio de caso, el cual según Ceballos Herrera (2009, p. 416) "[...] implica descripción, explicación y juicio. El estudio de casos es una modalidad de informe que valora la información para luego emitir un juicio" Los criterios para la escogencia de los casos fueron: Después de enfrentar a los estudiantes a varios problemas de aplicación, se revisaron los resultados y procesos llevados a cabo, y se seleccionaron los que presentaban más dificultades y que a la vez mostraron representaciones más claras, mejor escritura y mejor expresión verbal.

\section{Categorías}

En el Cuadro 1 se muestran las categorías de análisis con sus respectivos autores de referencia. 
Cuadro 1 - Categorías de análisis

\begin{tabular}{|l|l|l|}
\hline \multicolumn{1}{|c|}{ Categorías de análisis } & \multicolumn{1}{|c|}{ Subcategorías } & \multicolumn{1}{c|}{ Autor de referencia } \\
\hline Resolución de problemas & $\begin{array}{l}\text { - El conocimiento o recursos } \\
- \text { Estrategias cognitivas o heurísticas } \\
- \text { Las creencias y componentes afectivos }\end{array}$ & Schoenfeld (1992) \\
\hline Metacognición & Regulación (Planeación, Monitoreo y Evaluación) & Brown (apud TAMAYO, 2006) \\
\hline Medidas de tendencia central & Media, Moda y Mediana & $\begin{array}{l}\text { Batanero y Godino (2001), Carva- } \\
\text { lho (2001), Garrett y García Cruz } \\
\text { (2005) }\end{array}$ \\
\hline
\end{tabular}

Fuente: Elaboración del autor.

\section{Población}

La población de este estudio estuvo constituida por los estudiantes de grado $8^{\circ}$ de la Institución Educativa Normal Superior de Sincelejo-Sucre, Colombia, Grupos A y B, con un total de 83 estudiantes de género mixto, sus edades oscilan entre los 12 y 14 años. En el aspecto académico, el $25 \%$ de los estudiantes presentaron bajos resultados en el área de matemáticas en el año inmediatamente anterior; y no presentaban problemas disciplinarios.

\section{Muestra}

La muestra para esta investigación estuvo conformada por 6 estudiantes, todos ellos de genero mixto, (3 hombres y 3 mujeres), fueron elegidos mediante el muestreo no probabilístico de tipo intencionado, que según Sánchez y Reyes (2009) implica seleccionar la muestra representativa de la población según consideraciones específicas del investigador. Se escogieron estos estudiantes debido a que eran los que evidenciaban tener más dificultades en el área de matemáticas y bajo nivel de análisis en los procesos de resolución de problemas, y de esta manera poder verificar el impacto de la aplicación de la regulación metacognitiva para mejorar sus niveles de reflexión y comprensión. Así mismo, se seleccionó el tema de medidas de tendencia central, debido al bajo desempeño mostrado por los estudiantes en el pensamiento aleatorio y por la importancia que esta temática tiene en el campo de la estadística y su aplicación en la vida cotidiana.

\section{Técnicas e Instrumentos}

Las técnicas utilizadas en la investigación fueron: La observación participante, entrevista semi-estructurada, y el pensamiento en voz alta.

Se seleccionó la entrevista semiestructurada ya que según Hernández, Fernández y Baptista (2006, p. 597) "[...] se basan en una guía de asuntos o preguntas y el entrevistador tiene la libertad de introducir preguntas adicionales para precisar conceptos u obtener mayor información sobre los temas deseados". Es decir, no todas las preguntas están predeterminadas en la estructura de la entrevista. Cada una de las sesiones de entrevista y observación se grabaran con audio y video con el fin de poder realizar los reportes verbales de cada estudiante y el posterior análisis de la información recolectada. 
En lo referente a los instrumentos, su validez fue analizada mediante un estudio de expertos en el área de matemáticas y estadística de las diferentes instituciones educativas de la ciudad de Sincelejo, 15 docentes en total, de los cuales 10 son magister y 5 doctores. Estos instrumentos iban incluidos en una unidad didáctica y se aplicaron en diferentes momentos.

\section{Procedimiento}

Esta investigación se desarrolló en dos fases, en la primera se realizó la aplicación de una unidad didáctica, y en la segunda se realizó el análisis general de los resultados. La unidad didáctica se ejecutó durante 5 semanas, cuatro horas semanales para cada grupo, distribuidas así: (momento de ubicación, una semana, momento de desubicación, una semana y momento de reenfoque tres semanas). Esta unidad, contenía diferentes problemas sobre medidas de tendencia central, elaborados todos por el investigador. Los problemas planteados, permitían promover múltiples lenguajes y contenían preguntas de regulación metacognitiva.

En el Cuadro 2 se muestran algunos ejemplos de problemas realizados en cada momento y las respectivas preguntas metacognitivas.

Cuadro 2 - Ejemplos de problemas realizados en los diferentes momentos

\section{Momento de Ubicación - Problema 1}

La secretaría de educación Municipal de Sincelejo realizará unas olimpiadas de conocimientos por curso en los grados $8^{\circ}$, para poder participar se deben cumplir al menos 3 de las siguientes condiciones:

1. Que el promedio de notas del curso en el área de matemática sea mayor a 4.0.

2. Que la mediana de las edades sea 13 años

3. Que el peso promedio del curso sea mayor a $42 \mathrm{Kg}$

4. Que la moda de la variable género sea masculino

5. Que la mediana de la variable \# de hermanos sea 2

La Institución educativa Normal Superior de Sincelejo desea participar, por lo cual recolectó información en el grado $8^{\circ}$ que tiene 30 estudiantes, teniendo en cuenta las siguientes variables: edad (en años cumplidos), el género (femenino, masculino), el peso en Kg, número de hermanos y notas de los estudiantes en el área de matemática. Los datos recolectados fueron los siguientes:

a. Edades de los estudiantes: $12,15,12,13,13,14,13,12,14,13,15,14,14,13,15,14,14,14,14,13,13,15,16,14,15,13,15$, $16,14,15$

b. Género de los estudiantes: F, F, F, M, F, M, F, M, F, M, F, F, F, M, F, F, M, F, F, F, F, F, M, F, M, F, F, M, F, F

c. Peso en Kg: $35,40,35,41,35,38,40,38,41,38,40,38,42,38,40,38,41,46,47,38,43,42,38,41,38,41,42,43,45,39$

d. Notas de los estudiantes en el área de matemática: $3.5 ; 3.2 ; 4.4 ; 2.5 ; 3.6 ; 3.4 ; 4.2 ; 3.7 ; 2,8 ; 4,3 ; 3.9 ; 3.2 ; 4.1 ; 3.8 ; 3.2 ; 3.5 ; 5.0$; $3.6 ; 4.1 ; 3.9 ; 3.4 ; 3.5 ; 3.1 ; 3.7 ; 4.7 ; 4.9 ; 3.1 ; 3.6 ; 3.7 ; 4.3$.

e. Número de hermanos: 3, 1, 2, 4, 1, 0, 2, 1, 4, 2, 3, 3, 2, 1, 3, 5, 4, 5, 2, 1, 2, 2, 3, 1, 2, 4, 2, 6, 3, 1

¿Puede el grado $8^{\circ}$ de la Institución educativa Normal Superior de Sincelejo participar en las olimpiadas? ¿Por qué?

¿Qué entiendes por promedio? ¿Cuál es el peso promedio del curso?

¿Qué entiendes por moda? ¿Cuál es la Moda de la variable peso?

¿Qué entiendes por mediana? ¿Cuál es la mediana de la variable edades?

\section{Momento de Desubicación - Problema 2}

En Colombia y principalmente en Sincelejo hay un fenómeno que está creciendo cada vez más, el cual es el Mototaxismo, esto ha generado un aumento en el número de accidentes por día y el número de comparendos que se hacen debido al aumento de motos en la ciudad. Se preguntó en la oficina de tránsito el número de comparendos por día durante el mes de junio y estos fueron los resultados: $45,36,70,38,56,72,24,63,51,74,25,64,48,68,75,24,68,64,39,40,65,120,82,96,66$, $80,93,84,105,63$.

¿Cuáles es el número promedio de comparendos en el mes de junio?

¿Cuál es la mediana de los datos reportados por el tránsito?

Como el número de motos sigue en aumento, una empresa realizó una encuesta a 25 mototaxistas, para averiguar cuál es la marca de moto que más les gusta para trabajar: Auteco $(A)$, Yamaha ( $Y)$, Suzuki $(S)$ y Honda $(H)$, estas fueron sus respuestas: $A, S, A, Y, A, S, A, H, A, A, S, Y, Y, A, A, S, S, Y, A, S, Y, A, A, S, A$

¿Cuál es la moda en esta encuesta? ¿Por qué?

De igual manera, el número de accidentes ha aumentado durante los últimos meses, a continuación se muestra un diagrama del índice de accidentalidad durante los últimos 6 meses: 
\# de accidentes

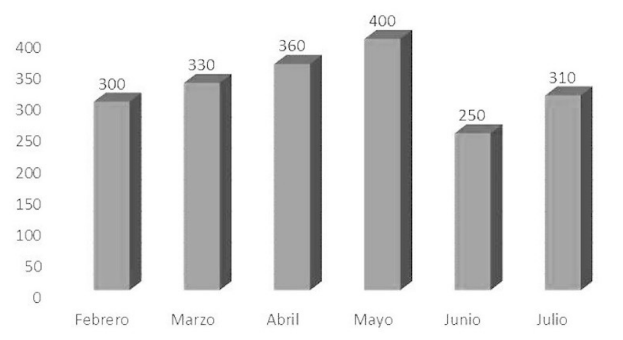

¿Cuál es el promedio de accidentes durante el último semestre?

¿Cuál es la moda en el diagrama?

¿Cuál es la mediana en el diagrama?

¿Cuál fue el promedio de accidentes por día durante el mes de abril?

Momento de Reenfoque - Problema 3

El grado $8^{\circ}$ quiere recolectar fondos para irse de excursión a fin de año, para esto ha decidido realizar actividades lucrativas para recolectar fondos. En la primera semana vende helados y las ventas fueron las siguientes:

$\begin{array}{lclc}\text { Días } & \text { \# de helados vendidos } & \text { sabores } & \text { \# de helados vendidos } \\ \text { Lunes } & 88 & \text { Piña } & 170 \\ \text { Martes } & 105 & \text { Vainilla } & 55 \\ \text { Miércoles } & 75 & \text { Chocolate } & 79 \\ \text { Jueves } & 84 & \text { Fresa } & 74 \\ \text { Viernes } & 98 & \text { Coco } & 72\end{array}$

¿Cuál fue el promedio de helados vendidos en la semana?, ¿Para qué le sirve esta información al curso?

¿Alrededor de cuantos helados deben traer para vender en la siguiente semana?, ¿Cuál es la moda en los datos de la tabla de sabores?

¿Para qué le sirve esta información al curso?, ¿De qué sabor deben traer más helados la siguiente semana? ¿Por qué?

\begin{tabular}{|c|c|c|}
\hline \multicolumn{3}{|c|}{ Preguntas Metacognitivas } \\
\hline \begin{tabular}{l}
\multicolumn{1}{c}{ Planeación } \\
¿Entendiste el enunciado del problema? \\
¿Cuántas veces lo leíste? \\
¿Subrayaste la información importante? \\
¿Organizaste los datos? \\
¿Cuál es la pregunta del problema? \\
¿Qué debes hacer para resolver el problema? \\
¿Tienes algún plan? Descríbelo
\end{tabular} & \begin{tabular}{l}
\multicolumn{1}{c}{ Monitoreo } \\
¿Crees que lo estás haciendo bien?: ¿Te sirve \\
el esquema que planteaste? \\
¿Estás revisando las operaciones? \\
¿Tienes alguna pregunta?
\end{tabular} & \begin{tabular}{l}
\multicolumn{1}{c}{ Evaluación } \\
¿Verificaste la solución? \\
¿Qué dificultades encontraste para resolver \\
el problema? \\
¿Qué fue lo que más le llamó la atención del \\
proceso realizado? ¿Crees que puedes realizar \\
problemas similares en menos tiempo? \\
¿Cómo te sentiste al resolver el problema? \\
¿Considera que el plan funcionó? Sí __ No \\
_Por qué?
\end{tabular} \\
\hline
\end{tabular}

Fuente: Elaboración del autor.

En la fase de aplicación de la unidad didáctica, se realizó el primer momento Ilamado de ubicación, en el cual se desarrolló el primer objetivo específico: Identificar los obstáculos ontológicos y procedimentales que presentan los estudiantes cuando solucionan problemas sobre medidas de tendencia central. Se tuvo en cuenta el discurso oral, escrito, gestos, imágenes, diagramas, entre otros, mediante el cual los estudiantes expresaron las respuestas de los problemas planteados. A partir de los resultados encontrados, se realizó una categorización de los obstáculos presentados, este análisis se mostró a los estudiantes, permitiendo que ellos identifiquen sus obstáculos iniciales.

En el segundo momento llamado de desubicación, se tuvieron en cuenta los obstáculos detectados y se hizo énfasis en la aplicación de estrategias de planeación, control y evaluación para resolver los problemas, Así mismo, se aplicaron dos problemas auténticos sobre medidas de tendencia central los cuales contenían preguntas de regulación metacognitiva que permitieron identificar al docente lo que iba sucediendo con los obstáculos caracterizados.

En el tercer momento llamado de reenfoque, se realizaron cuatro actividades con problemas auténticos sobre medidas de tendencia central. Durante los tres momentos se pudo identificar lo que sucedía con las categorías de análisis propuestas en la investigación, 
dicha exploración, permitió hacer comparación directa con lo realizado en los dos momentos anteriores, con el propósito de identificar en cada uno de los estudiantes cómo fue superando los obstáculos y analizando la manera cómo la metacognición contribuía a la resolución de problemas.

En la fase de análisis se desarrolló el segundo objetivo específico: Describir la relación que se da entre los procesos metacognitivos y las estrategias de resolución de problemas. Aquí se analizó la información recolectada en la aplicación de la unidad didáctica y se caracterizó de qué manera la reflexión metacognitiva favorece el proceso resolución de problemas sobre medidas de tendencia central. La información se organizó a través de una matriz, y después se asociaron las recurrencias dadas en las respuestas de los educandos con el fin de describir lo que sucedió en cada categoría.

\section{Análisis de datos}

El tipo de análisis que se realizó fue del discurso, según Tamayo et al. (2011, p. 96), "[...] el análisis del discurso, y por ende del lenguaje, permite acercarnos cualitativamente a diferentes representaciones de los estudiantes sobre distintos hechos o fenómenos."

Se realizó un análisis del discurso oral y escrito que realizaron los estudiantes en las diferentes actividades y en los diferentes momentos de la investigación. En este sentido Tamayo (2001, p. 48) manifiesta que "[...] el análisis de los textos escritos es usado ampliamente con el propósito de comprender los procesos de aprendizaje y cambio conceptual en los estudiantes". De igual manera, el análisis de los textos permitió obtener información de los obstáculos procedimentales y ontológicos que presentaban los estudiantes en la resolución de problemas sobre medidas de tendencia central. Así mismo, a través de los textos escritos se pudo verificar el avance de los educandos en todo el proceso.

\section{Resultados y Discusión}

Dentro de los obstáculos ontológicos presentados por los estudiantes en el momento de ubicación, en la resolución de problemas sobre medidas de tendencia central, se encontraron algunos relacionados con las concepciones inducidas. Estas concepciones están influenciadas por los procesos de socialización que se presentan en el ambiente escolar, y otras por la influencia de estos términos en el lenguaje cotidiano o uso común. En primer lugar, se muestran algunos ejemplos que proponen los estudiantes los cuales están relacionados con las notas que le entregan al final del periodo. De las 18 oraciones con sentido relacionadas con las concepciones inducidas todas estuvieron relacionadas con lo sociocultural (ver Cuadro 3).

Cuadro 3 - Concepciones acerca del promedio

\begin{tabular}{|c|l|}
\hline \multicolumn{1}{|c|}{ Pregunta } & \multicolumn{1}{c|}{ Respuesta del estudiante } \\
\hline P.1 ¿Qué entiendes por promedio? & E.1: Son las notas finales \\
& E.2: Es la suma de las notas del periodo \\
& E.3: Es la suma de las notas cuando me entregan el boletín \\
& E.4: Es la suma de las notas del área \\
& E.5: Es la suma de las notas finales \\
& E.6: Es la suma de las notas del periodo \\
\hline
\end{tabular}

Fuente: Elaboración del autor basada en registro de estudiantes. 
Todo lo anterior refleja que el contexto escolar de los estudiantes influye en su concepción acerca de lo que es el promedio, debido a que es en la escuela donde escuchan este término. En este sentido, Figueroa Molina, Utria Echeverría e Colpas Castillo (2006, p. 27) manifiestan que: "Los medios de comunicación, la interacción con amigos y familiares e incluso con los profesores constituyen una fuente de información de primera mano para los estudiantes. De allí obtienen ideas que fundamentan sus creencias y con las cuales forman sus conceptos sobre los fenómenos que observan."

Las respuestas dadas por los estudiantes manifiestan cierto obstáculo, debido a que asumen el promedio como una suma, olvidando que se tiene que dividir entre el total de los datos, lo cual ocasiona errores de procedimientos al momento de hallarlo. Otros elementos analizados en las medidas de tendencia central fueron la moda y la mediana, dentro de las cuales los estudiantes también presentaron concepciones inducidas (ver Cuadro 4).

Cuadro 4 - Concepciones acerca de la moda y mediana

\begin{tabular}{|l|l|}
\hline \multicolumn{1}{|c|}{ Pregunta } & \multicolumn{1}{|c|}{ Respuesta del estudiante } \\
\hline ¿Qué entiendes por moda? & E.1: Es algo que lo tiene todo el mundo. \\
& E.2: Es la ropa que se está usando \\
& E.3: Es lo que usa todo el mundo \\
& E.4: Es la ropa que usa la gente \\
& E.5: Es lo que se está usando en el momento \\
& E.6: Es lo que usa todo el mundo \\
\hline ¿Qué entiendes por mediana? & E.1: Es una persona pequeña de baja estatura. \\
& E.2: Es algo pequeño, pero no tanto. \\
& E.3: Es una forma de estatura pequeña \\
& E.4: Es una medida pequeña, no tan grande \\
& E.5: Es un valor muy pequeño \\
& E.6: Es un valor pequeño que va en el centro \\
\hline
\end{tabular}

Fuente: Elaboración del autor basada en registro de estudiantes.

En las respuesta de los estudiantes se presentan concepciones inducidas, influenciadas por el entorno social y cultural inmediato donde se expone el termino moda y mediana, asociando el termino al lenguaje cotidiano, intentando de forma más o menos consciente transferir el significado que se le da habitualmente a su significado en el campo de la estadística. Todo lo anterior refleja la influencia del contexto inmediato del estudiante en la formación de los conceptos, en este sentido, Pozo et al. (1991, p. 88) manifiesta: "[...] entre las fuentes socioculturales del conocimiento del alumno cabe destacar no solo la familia y el sistema educativo sino también la creciente influencia de los medios de comunicación en la formación de concepciones".

Dentro de los obstáculos procedimentales presentados por los estudiantes en la resolución de problemas sobre medidas de tendencia central, está escoger como el promedio el dato mayor, siendo este un valor que les llama la atención dentro de la muestra o posiblemente tomaron un valor cualquiera para dar respuesta a lo pedido. En este sentido, Garrett y García Cruz (2005) señalan que dentro de las estrategias incorrectas en el cálculo de la media está elegir un dato cualquiera como media, para la solución pretendida. Otros se limitaron a sumar los datos olvidando que se debe dividir entre el total.

Del mismo modo los estudiantes presentaron el obstáculo de creer que la moda era el dato mayor y no el de mayor frecuencia. En este sentido Carvalho (2001) manifiesta que dentro de los errores que presentan los estudiantes en el cálculo de la moda, está tomar la 
mayor frecuencia absoluta, en lugar de tomar el valor de la variable que aparece con mayor frecuencia. Subyace en este error una confusión entre frecuencia y valor de la variable.

Frente a la subcategoría mediana, los estudiantes escogieron el valor del centro, olvidando ordenar los datos, por lo cual presenta un error al hallar la mediana, en este sentido Carvalho (2001) manifiesta que dentro de los errores que presentan los estudiantes en el cálculo de la mediana está no ordenar los datos para calcular la mediana, por entender la mediana como el centro "no ordenado" de la distribución. De igual manera, otro de los errores procedimentales recurrentes es asumir la mediana como el valor más pequeño.

Dentro de los errores procedimentales más notorios en la resolución de problemas sobre medidas de tendencia central se encuentran: manejo inadecuado de las propiedades de las medidas de tendencia central, aplicar procedimientos incorrectos, confundir las medidas de tendencia central, no contar con un plan de ejecución, no ordenar los datos, baja comprensión lectora, repetir algoritmos de forma mecánica, dejando a un lado la comprensión de los conceptos y el análisis de lo que se está haciendo.

Teniendo en cuenta que en el momento de ubicación los procesos de regulación metacognitiva no eran llevados a cabo por los estudiantes, se realizó la explicación por parte del docente del objetivo de las preguntas en la planeación, monitoreo y evaluación, aspecto que empezó a llevarse a cabo y a mejorar desde el segundo momento (desubicación), permitiendo optimizar la capacidad de resolver problemas sobre medidas de tendencia central de forma gradual. A continuación se describen los aspectos que se permitieron optimizar en los estudiantes.

Desde el momento de desubicación hasta el de reenfoque, la planeación como primer proceso que se lleva a cabo al resolver el problema, permitió que los estudiantes diseñaran una serie de pasos a través de los cuales iban a realizar cada una de las actividades del problema. En primer lugar, realizaban la lectura comprensiva del problema, después de entenderlo y saber cuál era la pregunta, se presentaba la organización de los datos, seguidamente se identificaban las fórmulas para aplicar y posteriormente realizaban las operaciones pertinentes. Dentro de las estrategias aplicadas se destacan: leer varias veces el problema, encerrar en círculos datos importantes, utilizar líneas para resaltar números, organizar los datos en forma ascendente, extraer datos de los diagramas e identificar las operaciones a utilizar. Es decir, entre más estrategias cognitivas tenga el educando mejor puede ser su proceso de planeación. En este sentido, Schoenfeld (1992) manifiesta que las estrategias cognitivas o heurísticas involucran formas de representar y explorar los problemas con la intención de comprender los enunciados y plantear caminos de solución.

En el cuadro 5 se muestra las estrategias cognitivas utilizadas por los estudiantes.

Cuadro 5 - Estrategias cognitivas

\begin{tabular}{|c|l|}
\hline \multicolumn{1}{|c|}{ Procedimiento } & \multicolumn{1}{c|}{ Estrategia } \\
\hline$\overline{\mathrm{A}}, \overline{\mathrm{S}}, \overline{\mathrm{A}}, \overline{\mathrm{H}}, \overline{\mathrm{A}}, \overline{\mathrm{A}}, \overline{\mathrm{S}}, \overline{\mathrm{Y}}, \overline{\mathrm{Y}}, \overline{\mathrm{A}}, \overline{\mathrm{A}}, \overline{\mathrm{S}}, \overline{\mathrm{S}}, \overline{\mathrm{Y}}, \overline{\mathrm{A}}, \overline{\mathrm{S}}, \overline{\mathrm{Y}}, \overline{\mathrm{A}}, \overline{\mathrm{A}}$, & $\begin{array}{l}\text { Realizar marcas encima de los datos } \\
\text { (Realizado en Problema 2) }\end{array}$ \\
\hline $\begin{array}{l}\text { a) Edades de los estudiantes: } \\
12,15,12,13,13,14,13,12,14,13,15.14,14,13,15,14,14,14,14,13,13,15, \\
16,14,15,13,15,16,14,15\end{array}$ & $\begin{array}{l}\text { Encerrar los datos con círculos o } \\
\text { cuadrados. (Realizado en Problema 2) }\end{array}$ \\
$75,84,88,98,105$ & $\begin{array}{l}\text { Organizar en forma ascendente los } \\
\text { datos de una tabla (Realizado en } \\
\text { problema 3) }\end{array}$ \\
\hline
\end{tabular}




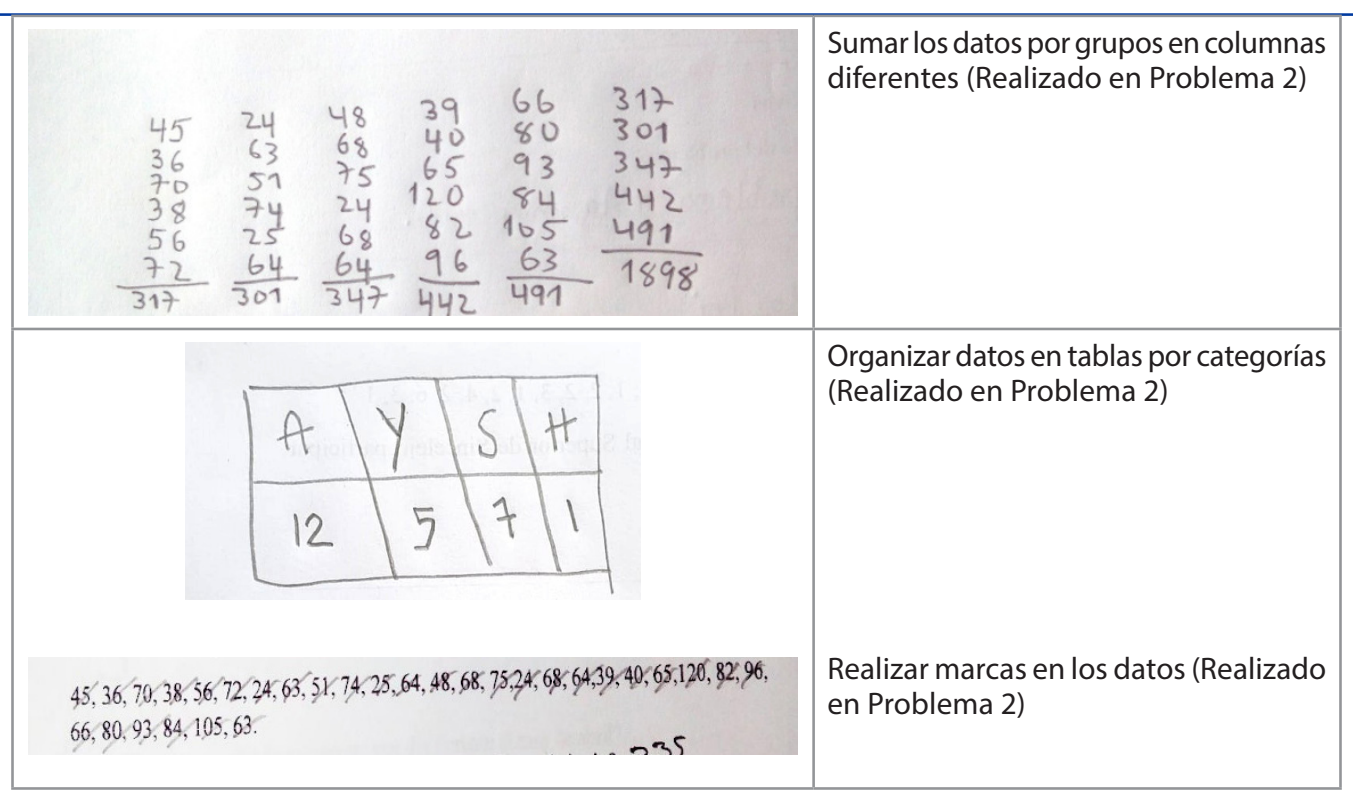

Fuente: Elaboración del autor basado en registro de estudiantes.

En cuanto al monitoreo, desde el momento de desubicación hasta el de reenfoque, permitió a los estudiantes reflexionar si lo que estaban haciendo estaba bien, revisar operaciones, corregir errores, leer nuevamente el problema y replantear estrategias de cálculo. También pudieron reconocer los aciertos y dificultades durante el proceso. A continuación se presentan algunas respuestas de los estudiantes en el proceso de monitoreo.

Los estudiantes E.1, E.2, E.4 manifiestan: "a veces me devuelvo a leer nuevamente el problema varias veces". El estudiante E.3 expresa: "al revisar la división en el promedio, me di cuenta que tenía un error, borré lo que tenía malo y lo corregí". El estudiante E.5 expresa: "rectifiqué la suma al hallar el promedio y tenía un error que después corregí". El estudiante E.6 manifiesta: "en la mediana se me había olvidado ordenar los datos y rectifiqué y los ordené y después resolví el problema", (respuestas dadas en el momento de desubicación).

En las respuestas dadas por los estudiantes, se evidencia que realizaron monitoreo del proceso llevado a cabo, revisando las operaciones para verificar si estaban bien, contaban varias veces los datos, utilizaron marcas en los números y algunas veces borran para corregir operaciones. Se evidencia más comprensión del tema y verifican mejor lo que están haciendo. Al respecto, Sanz (2010, p. 116) plantea que el monitoreo "[...] consiste en la realización de la actividad y en el control que se ejerce sobre cada uno de los aspectos implicados en su desarrollo y sobre los posibles factores que pueden incidir en la concentración y distribución de los recursos".

En cuanto a la evaluación, desde el momento de desubicación hasta el de reenfoque, permitió a los educandos saber si se cumplió el objetivo propuesto e identificar algunas dificultades en el proceso llevado a cabo, además, se pudo evaluar la efectividad de los pasos realizados. A continuación se presentan algunas respuestas de los estudiantes frente al proceso de evaluación en el momento de reenfoque.

Los estudiantes E.1, E.2; responden: "una de las dificultades que tuve en el problema fue organizar los datos para hallar el promedio", "corregí una de las sumas, ya que me había equivocado", "el plan que diseñé me sirvió porque esos fueron los pasos y me funcionaron", E.3, E.4; manifiestan: "El plan que diseñé me sirvió porque fui realizando el problema lentamente y esto me permitió corregir algunas cosas", E.5, expresa: "después que terminé el problema me dediqué a revisar cada una de las operaciones para ver si estaban bien" y E.6 manifiesta: "menos mal que revisé porque tenía un error en la división cuando hallé el promedio". 


\section{Discusión}

En los procesos de resolución de problemas, es importante reconocer los obstáculos ontológicos y procedimentales que los estudiantes presentan, debido a que esto permite tener una visión clara y real del estado de los educandos lo cual se puede lograr a través de preguntas metacognitivas, en este sentido, se confirma lo que manifiesta Tamayo et al. (2011, p. 119) "[...] la práctica de la metacognición facilita la identificación de obstáculos epistemológicos, lingüísticos y pedagógicos en los actores del proceso de enseñanzaaprendizaje."

En lo referente a la identificación de los obstáculos que presentan los estudiantes al momento de resolver problemas, el principal obstáculo procedimental que se presenta en el uso de la mediana, es que los estudiantes no ordenan los datos, en la media aritmética no dividen por el total de los datos, y en la moda escogen el dato mayor y no el de mayor frecuencia, lo cual coincide con las investigaciones de Carvalho (2001), Cobo (2003) y Mayen Galicia (2009).

Uno de los aportes de esta investigación, es que permitió revelar que los obstáculos ontológicos y procedimentales que presentan los estudiantes, se pueden detectar con la aplicación de la regulación metacognitiva como estrategia, y para su implementación el proceso debe ser guiado por el docente, teniendo en cuenta las fortalezas y limitaciones de los educandos.

Se plantea como sugerencia diseñar otro tipo de investigaciones, en la cual se apliquen nuevas estrategias metacognitivas en la resolución de problemas en otras temáticas y áreas, de tal manera que se pueda contribuir a la formación de estudiantes autónomos y conscientes de sus fortalezas y debilidades.

\section{Conclusiones}

La regulación metacognitiva permite a los estudiantes mejorar los procesos de resolución de problemas. Los educandos objeto de estudio, después de la intervención, son capaces de aplicar las tres categorías: planear, controlar y evaluar. Además, reflexionan más acerca de los procesos que llevan a cabo. La dificultad de su implementación, se muestra cuando los estudiantes presentan obstáculos procedimentales que le impiden avanzar.

En lo referente a la planeación como actividad previa a la solución de los problemas, permite el diseño de unas estrategias que incluyen las acciones a seguir, en este proceso se tiene en cuenta el conocimiento que posee el estudiante acerca del tema, sus dificultades y los recursos cognitivos con que cuenta para enfrentar los problemas, y de esta manera realizar una serie de pasos organizados para poder resolver el problema planteado.

Así mismo, el control como proceso que se realiza desde el instante en que se inicia la ejecución de las acciones, permite la verificación de las actividades que se van realizando, en este caso, las operaciones y rectificación de las mismas. Esto implica, la revisión de la estrategia empleada e identificar si se está llevando a cabo el plan que se diseñó. En algunos casos, el estudiante puede transformar el plan y sus estrategias, organizando nuevamente los datos y rectificando las operaciones de diferentes maneras, aspecto que se presentó en 5 de los 6 estudiantes objeto de estudio. 
Del mismo modo, la evaluación como actividad que ayuda a contrastar los resultados con lo que se planea al principio, permite la valoración de los resultados de la estrategia utilizada, como también la rectificación de algunas de las operaciones que se llevan a cabo en todo el proceso. Esto conlleva a corregir algunos pasos y replantear en algunas ocasiones lo que se hizo, aspecto que se presentó en 5 de los 6 estudiantes objeto de estudio.

En lo referente a las dimensiones que explican el éxito o fracaso de los estudiantes en la resolución de problemas, los recursos, permiten al docente saber cuáles son los conceptos, fórmulas y fundamentos con los que cuenta el estudiante acerca de las medidas de tendencia central. Las estrategias cognitivas, permiten al docente saber las formas en que los educandos representan y exploran los problemas, cómo comprenden los enunciados y cómo plantean los caminos de solución, y en lo referente a las creencias, permiten al docente saber la conceptualización del estudiante acerca de la resolución de problemas, y la actitud y disposición para trabajar. De los 6 estudiantes objeto de estudio 5 mejoraron el nivel en los procesos de resolución de problemas y las categorías que más incidieron en esto fueron la planeación y el monitoreo, solo uno de ellos permaneció con algunas dificultades asociadas a errores procedimentales.

Otro aspecto importante de la investigación, es que se pudo reconocer que la regulación metacognitiva por sí sola no permite mejorar los procesos de resolución de problemas, se necesita el conocimiento del tema y el manejo de los aspectos procedimentales.

Los obstáculos ontológicos más frecuentes presentados en los 6 estudiantes frente a los conceptos sobre medidas de tendencia central fueron: Considerar la media aritmética solo como una suma de notas, la mediana como el dato más pequeño (estatura baja) y la moda como lo que se está usando en el momento. Todo lo anterior se manifiesta como concepciones inducidas por el entorno social y cultural inmediato del estudiante. Todos estos obstáculos fueron evidenciados en el primer momento (ubicación) y fueron desapareciendo gradualmente con la implementación de la reflexión metacognitiva desde el segundo momento.

\section{Referencias}

BATANERO, C.; GODINO, J. D. Análisis de datos y su didáctica. Granada: Universidad de Granada, 2001.

CARVALHO, C. F. Interacção entre pares: contributos para a promoção do desenvolvimento lógico e do desempenho estatístico, no $7^{\circ}$ ano de escolariedade. 2001. Tesis (Doctorado en Educación) Universidade de Lisboa, Lisboa, 2001.

CEBALLOS HERRERA, F. El informe de investigación con estudio de casos. Magis: revista internacional de investigación en educación, Bogotá, v. 1, n. 2, p. 413-423, 2009.

COBO, B. Significados de las medidas de posición central para los estudiantes de secundaria. 2003. Tesis (Doctorado de Didáctica de la Matemática) - Universidad de Granada, Granada, 2003.

CORTÉS CORTÉS, M.; IGLESIAS LEÓN, M. Generalidades sobre metodología de la investigación. México: Universidad Autónoma del Carmen, 2004.

FIGUEROA MOLINA, R.; UTRIA ECHEVERRÍA, C.; COLPAS CASTILLO, R. Entendimiento conceptual de los estudiantes del nivel de básica secundaria sobre el concepto de ácido. Tecné, Episteme, Didaxis, Bogotá, v. 19, p. 22-31, 2006. DOI: https://doi.org/10.17227/ted.num19-1040. 
HERNÁNDEZ, R.; FERNÁNDEZ, C.; BAPTISTA, L. P. Metodología de la investigación. México: McGraw Hill Interamericana, 2006.

KLINGLER, C. Psicología cognitiva: estrategias en la práctica docente. México: McGraw Hill, 2001.

LESH, R.; ZAWOJEWSKI, J. S. Problem solving and modeling. In: LESTER JR., F. K. (ed.). The second handbook of research on mathematics teaching and learning. Charlotte: Information Age Publishing, 2007. p. 763-804.

MAYEN GALICIA, S. A. Comprensión de las medidas de tendencia central en estudiantes mexicanos de educación secundaria y bachillerato. 2009. Tesis (Doctorado de Didáctica de la Matemática) Universidad de Granada, Granada, 2009.

MATO-VÁZQUEZ, D.; ESPIÑEIRA, E.; LÓPEZ-CHAO, V. A. Impacto del uso de estrategias metacognitivas en la enseñanza de las matemáticas. Perfiles Educativos, Mexico, v. 39, n. 158, p. 91-111, 2017.

MORENO CASTIBLANCO, A. N.; DAZA TORRES, B. Y. Incidencia de estrategias metacognitivas en la resolución de problemas en el área de la matemática. 2014. Trabajo de Grado (Maestría en Educación) - Pontificia Universidad Javeriana, Bogotá, 2014.

ORTIZ BUENO, S. X.; VALENCIA CASTRILLÓN, A. V. Conocimiento metacognitivo en estudiantes de básica primaria. 2017. Tesis de Grado (Maestría en Educación y Desarrollo Humano) - Universidad de Manizales, Manizales, 2017.

ORTON, A. Didáctica de las matemáticas. Madrid: MEC-Morata, 1990.

POZO, J. A.; SANZ, A.; GÓMEZ CRESPO, M. A.; LIMÓN, M. Las ideas de los alumnos sobre las ciencias: una interpretación desde la psicología cognitiva. Enseñanza de las Ciencias, Barcelona, v. 9, n. 1, p. 83-94, 1991.

PULIDO, L. M. Procesos metacognitivos que llevan a cabo estudiantes de grado noveno con desempeños superior y bajo del colegio Agustín Fernández I. E. D. durante la resolución de problemas matemáticos. Trabajo de grado (Maestría en Educación) - Pontificia Universidad Javeriana, Bogotá, 2014.

QUINTERO GARCÍA, E. A. Dificultades que identifican los estudiantes a través de la metacognición en el aprendizaje de las matemáticas en educación secundaria. 2014. Tesis (Magíster en Enseñanza de las Ciencias) - Universidad Autónoma de Manizales, Manizales, 2014.

SÁNCHEZ, H.; REYES, C. Metodología y diseño en la investigación científica. Lima: Visión Universitaria. 2009.

SANZ, M. Competencias cognitivas en educación superior. Madrid: Narcea, 2010.

SCHOENFELD, A. H. Learning to think mathematically: problem solving, metacognition, and sense making in mathematics. In: GROUWS, D. A. (ed.). Handbook of research on mathematics teaching and leaming. Reston: NCTM, 1992. p. 334-370.

TAMAYO, O. Evolución conceptual desde una perspectiva multidimensional: aplicación al concepto de respiración. 2001. Tesis (Doctorado de Didáctica de la Matemática y de las Ciencias Experimentales) - Universidad Autónoma de Barcelona, Barcelona, 2001. Recuperado el 18 ene. 2017 de: https://ddd.uab.cat/record/37624.

TAMAYO, O. La metacognición en los modelos para la enseñanza y el aprendizaje de las ciencias. In: Los bordes de la pedagogía: del modelo a la ruptura. Bogotá: Universidad Pedagógica Nacional, 2006. p. 275-306.

TAMAYO, O.; VASCO, C. E.; GARCÍA, L. I.; GIRALDO, A.; RIVERO, M.; QUICENO, H.; SUAREZ, M. M. La clase multimodal y la formación y evolución de conceptos científicos a través del uso de tecnologías de la información y la comunicación. Manizales: Universidad Autónoma de Manizales, 2011. 